\title{
Gerechtigkeit in flexiblen Arbeits- und Managementprozessen
}

\author{
Gregor Engels, Günter W. Maier, Sonja K. Ötting, Eckhard Steffen \\ und Alexander Teetz
}

\subsection{Einleitung}

Der Wandel von nationalen Industrie- zu globalen Informationsgesellschaften führt in der industriellen Produktion zu nahezu epochalen Veränderungen, die häufig als vierte industrielle Revolution bezeichnet werden. Unter dem Schlagwort „Industrie 4.0“ (Kagermann et al. 2011) werden neu entstehende Smart Factories verstanden, in denen bereichs- und unternehmensübergreifende Leistungserbringungsprozesse durchgängig durch informationstechnische Systeme unterstützt werden.

G. Engels $(\varangle) \cdot$ A. Teetz

Institut für Informatik, Universität Paderborn, Zukunftsmeile 1

33102 Paderborn, Deutschland

e-mail: engels@uni-paderborn.de; alexander.teetz@uni-paderborn.de

G.W. Maier

Abteilung für Psychologie, Universität Bielefeld, Postfach 1001 31,33501 Bielefeld, Deutschland

e-mail: g.maier@uni-bielefeld.de

S.K. Ötting

Abteilung für Psychologie und CoR-Lab, Universität Bielefeld

Postfach 1001 31, 33501, Bielefeld, Deutschland

e-mail: soetting@uni-bielefeld.de

E. Steffen

Paderborn Center for Advanced Studies, Universität Paderborn

Fürstenallee11, 33102, Paderborn, Deutschland

e-mail: eckhard.steffen@uni-paderborn.de 
Weniger revolutionär gesehen sind Smart Factories das Ergebnis eines evolutionären Veränderungsprozesses. Vorläufer von gegenwärtigen und zukünftigen Prozessunterstützungssystemen mit geringerer Komplexität wurden bereits intensiv untersucht (Radhakrishnan et al. 2011). Hierzu zählen etwa Systeme zur digitalen Konstruktion (CAE Computer-Aided Engineering, CAD - Computer-Aided Design), zur Arbeitsplanung (CAP - Computer-Aided Planning) oder zur Fertigung (CAM - Computer-Aided Manufacturing). Durch die Hinzunahme betriebswirtschaftlicher Produktionsplanungs- und Produktionssteuerungssysteme entstanden Systeme zum Computer-Integrated Manufacturing (CIM), Computer-Integrated Business (CIB) bzw. Leitstände (Production Control Systems). Im Bereich der betrieblichen Informationssysteme wurden Unterstützungssysteme zur Geschäftsprozessmodellierung und -ausführung (BPM - Business Process Management) untersucht (Weske 2012) und im Bereich der Arbeitsunterstützungssysteme Workflow-Management-Systeme (van der Aalst 2013).

Smart Factories unterscheiden sich von den oben genannten Unterstützungssystemen durch eine völlig neue Produktionslogik. Die eingebetteten Produktionssysteme sind vertikal mit betriebswirtschaftlichen Prozessen innerhalb von Fabriken und Unternehmen vernetzt und horizontal zu verteilten, in Echtzeit steuerbaren Wertschöpfungsnetzwerken verknüpft - von der Konzipierung eines Produktes bis zur Ausgangslogistik. Gleichzeitig ermöglichen und erfordern sie ein durchgängiges Engineering über die gesamte Wertschöpfungskette hinweg. Basis und kennzeichnend für Smart Factories (Industrie 4.0) sind Cyber-Physical Systems (CPS), die Vernetzung physikalischer Systeme wie Produktionsmaschinen und Transportsysteme mit einer durch Software geprägten weitläufig verteilten Rechner- und Netzinfrastruktur, die für Steuerung und Kontrolle der Ablaufe zuständig ist. Den Endgeräten, die die Schnittstelle zwischen der realen, physikalischen Welt und dem Internet realisieren, kommt dabei eine besondere Bedeutung zu. Sie werden in Anlehnung an den Begriff Physical Device als Cyber-Physical Devices (CPD) bezeichnet (Kleinjohann et al. 2013).

Cyber-Physical Systems und insbesondere Cyber-Physical Devices interagieren mit den Beschäftigten und können dabei flexibel und leistungsfähig in den Produktionsprozess integriert werden. Für die Interaktion von technischen und menschlichen Akteuren können unterschiedliche Benutzungsschnittstellen verwendet werden. Neben etablierten Multi-Touch Displays sind auch haptische sowie auf Tiefenkameras basierende berührungslose Schnittstellen mit mechanischem bzw. auditivem Feedback verfügbar. Diese Schnittstellen erlauben eine direkte Mensch-Maschine-Interaktion. Weiterhin haben CPDs die Eigenschaft, mit Hilfe von Sensordaten auf veränderte Rahmenbedingungen zu reagieren. Dies impliziert, dass Entscheidungsprozesse, wie z. B. Bestellungen von Waren oder Entscheidungen über den Einsatz von Personal (z. B. KapaflexCy; Kapazitätsflexibilität in Cyber-Physical Systems (Bauer et al. 2014)) automatisch durch ein den Produktionsprozess steuerndes System initiiert und verbindlich ausgelöst werden. An diesen Beispielen wird deutlich, dass Entscheidungen, die häufig auf Geschäftsführungs- bzw. Managementebene angesiedelt sind, mittlerweile bereits und in Zukunft noch mehr durch ein technisches System getroffen werden. Dementsprechend sind Beschäftigte, die innerhalb von 
Produktionsumgebungen mit technischen Unterstützungssystemen arbeiten, bei hinreichender Komplexität des Unterstützungssystems von „Entscheidungen“ dieses Systems betroffen. Hier stellt sich die Frage, wie die Menschen solche Entscheidungen wahrnehmen und unter welchen Umständen sie bereit sind, sie zu akzeptieren und sie als angemessen und gerecht zu beurteilen.

Die mit der Digitalisierung erreichte Flexibilisierung der Arbeitsprozesse erlaubt einem Unternehmen einerseits schnell auf veränderte Markt- bzw. Rahmenbedingungen zu reagieren, andererseits verändern sich dadurch auch die Arbeits- und Lebensbedingungen der betroffenen Beschäftigten. Wenn es um die Integration von Beschäftigen in die digitale Arbeitswelt geht, müssen schon im Planungsprozess die Bedarfe von Beschäftigten vollumfänglich berücksichtigt werden. Nur so kann sichergestellt werden, dass die neuen Prozesse und technischen Möglichkeiten erfolgreich angewendet und genutzt werden. Ein Aspekt, der schon bei der Prozessmodellierung berücksichtigt werden sollten, ist beispielsweise neben den Kriterien Ausführbarkeit, Schädigungslosigkeit, Beeinträchtigungsfreiheit und Persönlichkeitsförderlichkeit von Arbeit (Hacker 1984; Hacker und Richter, 1984) auch die Frage, in welchem Ausmaß Entscheidungen des technischen Systems als gerecht wahrgenommen werden. Gerade die Wahrnehmung organisationaler Gerechtigkeit beeinflusst zentrale Arbeitseinstellungen und -verhalten (Colquitt et al. 2001, 2013).

Im weiteren Verlauf dieses Kapitels werden zunächst in der Literatur dargestellte Gerechtigkeitsaspekte bei Entscheidungen in Produktionsprozessen vorgestellt. Nachfolgend wird ein Konzept zur Integration von Gerechtigkeitsaspekten in derartige Entscheidungsprozesse auf Basis existierender Prozessnotationen vorgestellt. Das Kapitel schließt mit Handlungsempfehlungen in Bezug zur Integration von Gerechtigkeitsaspekten in Entscheidungsprozesse von automatisierten Produktionsabläufen.

\subsection{Gerechtigkeitsaspekte in Entscheidungsprozessen von automatisierten Produktionsabläufen}

Vielfältige arbeits- und organisationspsychologische Forschung zeigt, dass die Wahrnehmung von Fairness durch die Prinzipien organisationaler Gerechtigkeit bestimmt wird (für einen Überblick siehe z. B. (Colquitt 2001)). Die organisationale Gerechtigkeit hat sich im Laufe der Forschung als eine der wichtigsten Determinanten des Erlebens und Verhaltens von Beschäftigten in Organisationen gezeigt. Hier zeigten sich unter anderem positive Zusammenhänge zu Arbeitszufriedenheit, organisationalem Commitment, Arbeitsleistung und Organizational Citizenship Behavior sowie negative Zusammenhänge zu kontraproduktivem Arbeitsverhalten (im Überblick z. B. in (Colquitt et al. 2001) und (Colquitt et al. 2013)). Die Prinzipien organisationaler Gerechtigkeit setzen sich aus den in Abb 16.1 dargestellten und im Folgenden beschriebenen vier Dimensionen zusammen (Colquitt und Zipay 2015). 


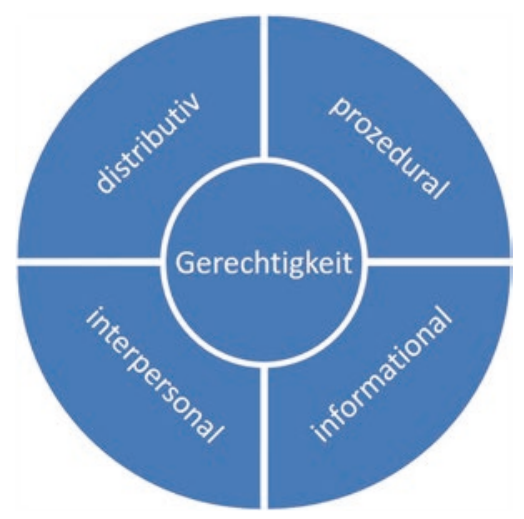

Abb. 16.1 Prinzipien der Gerechtigkeit

Distributive Gerechtigkeit, oder auch Verteilungsgerechtigkeit, bezieht sich auf die Gerechtigkeit der Ergebnisse einer Entscheidung. Das Ergebnis einer Entscheidung wird z. B. dann als gerecht wahrgenommen, wenn das eigene Verhältnis von Input zu Output dem einer Vergleichsperson entspricht.

Prozedurale Gerechtigkeit beschreibt die Gerechtigkeit des Entscheidungsprozesses. Eine Entscheidung wird dann als prozedural gerecht wahrgenommen, wenn der Entscheidungsprozess zum einen eine Möglichkeit der Mitsprache („,voice“) beinhaltet. Zum anderen sollte ein gerechter Entscheidungsprozess konsistent über Zeit und Personen unvoreingenommen und akkurat sein, eine Korrekturmöglichkeit enthalten und ethischen und moralischen Standards genügen.

Die letzten beiden Dimensionen betreffen die Gerechtigkeit der Kommunikation einer Entscheidung.

Interpersonale Gerechtigkeit bezieht sich auf das Verhalten des Entscheidungsträgers gegenüber dem Entscheidungsempfänger. Eine Entscheidung wird dann als interpersonal gerecht wahrgenommen, wenn sie respektvoll und höflich kommuniziert wird.

Informationale Gerechtigkeit bezieht sich auf die Angemessenheit der Informationen und Erklärungen darüber, wie der Entscheidungsprozess gestaltet ist. Eine Entscheidung wird dann als informational gerecht wahrgenommen, wenn die Erklärungen ehrlich, begründet, spezifisch und zeitnah kommuniziert werden.

Eine Entscheidung wird dann als fair wahrgenommen, wenn die Prinzipien organisationaler Gerechtigkeit erfüllt sind. Bisher wurden Gerechtigkeitswahrnehmungen fast ausschließlich in der Mensch-Mensch-Interaktion untersucht (für eine Ausnahme siehe (Shank 2012, 2014)). Es gibt jedoch erste Überlegungen, wie Prinzipien der Gerechtigkeit auch in die Mensch-System-Interaktion übertragen werden können (vgl. Töniges et al. 2017). Hierbei wird es wichtig sein, Prinzipien der Gerechtigkeit sowohl in der Entwicklung als auch im Betrieb von Cyber-Physical Systems entsprechend zu berücksichtigen. Daher wird im nächsten Abschnitt dargestellt, wie Gerechtigkeitsaspekte in der in Cyber-Physical Systems enthaltenen Prozess- und Entscheidungsunterstützung berücksichtigt werden können. 


\subsection{Prozessunterstützung}

In diesem Abschnitt wird auf die modellbasierte Entwicklung einer Prozess- und Entscheidungsunterstützung unter Berücksichtigung der im vorherigen Abschnitt beschriebenen Gerechtigkeitsaspekte eingegangen. Hierzu werden zunächst die vier Phasen (1) Entwurf \& Analyse, (2) Konfiguration, (3) Enactment und (4) Evaluierung eines Prozessmanagement-Lebenszyklus näher betrachtet (Weske 2012). Der Fokus liegt hierbei insbesondere auf ausgesuchten Prozessaspekten, die bei der Realisierung von gerechten Abläufen für Industrie 4.0-Anwendungen erforderlich sind.

In der Phase (1) Entwurf \& Analyse werden Prozessmodelle für Produktions- und Entscheidungsprozesse unter Einsatz einer Prozessmodellierungssprache erstellt. Hierbei sollten strategische oder taktische Vorgaben oder Leitlinien etwa zur Gestaltung von Entscheidungsprozessen (auch unter Einbeziehung von Gerechtigkeitsaspekten) in Produktionsabläufen berücksichtigt werden. In einer anschließenden Analyse können Eigenschaften der erstellten Prozessmodelle überprüft werden. Hierzu können ebenso Fragen der modellierten Gerechtigkeit gehören.

Die Phase (2) Konfiguration bezieht sich auf die Vorbereitung der Ausführung der in der vorherigen Phase entwickelten Modelle. Hierzu gehört insbesondere auch die (gerechte) Planung und Zuteilung von Ressourcen, wie z. B. konkrete Produktionszeiträume, Schichtpläne oder die Auswahl von Beschäftigten.

Die Bildung von konkreten Prozessinstanzen und deren Ausführung findet in der Phase (3) Enactment statt. In dieser Phase finden operative Interaktionen von Akteuren bzw. Nutzern mit Systemen statt. Im Rahmen dieser Interaktionen werden sowohl von maschineller als auch von menschlicher Seite Instanzen von Entscheidungsprozessen durchlaufen, die auch die genannten Gerechtigkeitsaspekte betreffen können. Ferner können im laufenden Betrieb durch Feedback-Mechanismen Informationen zur Gerechtigkeitsempfindung erfasst, automatisch analysiert und zur Berücksichtigung - bei späteren Entscheidungen - gespeichert werden.

Die Phase (4) des Lebenszyklusmodells befasst sich mit der Analyse aktiver und beendeter Prozessinstanzen zwecks Evaluierung. Ein Ergebnis dieser Phase können Anforderungen sein, die im Rahmen der Prozessverbesserung, etwa auch einer verbesserten Gerechtigkeitsunterstützung, umgesetzt werden.

Grundlage für alle vier Phasen eines Prozessmanagement-Lebenszyklus ist der Einsatz einer Prozessmodellierungssprache wie z. B. BPMN (Business Process Modeling and Notation (Großkopf et al. 2009)). Wesentliche Aspekte einer solchen Modellierungssprache betreffen auch die Modellierung und Umsetzung von Gerechtigkeitsaspekten.

Sequentielle Abläufe stellen die einfachste Form einer möglichen Ablaufstruktur in einem Prozessmodell dar. Daneben sind komplexe Aufgaben aber häufig durch Arbeitsabläufe beschrieben, die neben Verzweigungen und Alternativen auch den Aufruf von Unterprozessen enthalten können. In diesem Zusammenhang spricht man auch von hierarchischen Ablaufstrukturen. Bei Verwendung all dieser Sprachelemente in einem 
Prozessmodell stellt sich für den Modellierer stets die Frage nach einer adäquaten Berücksichtigung von Gerechtigkeitsaspekten.

Darüber hinaus sind neben sequentiellen oder hierarchischen Abläufen im Umfeld von Industrie 4.0-Anwendungen insbesondere auch vernetzte Abläufe existent. In vernetzten Abläufen kommunizieren vernetzte Partner miteinander über ein oder mehrere Medien. Für die Prozessmodellierung bedeutet dieser Umstand die Notwendigkeit der Modellierung der Kommunikation als Ganzes - dies umfasst sowohl die Kommunikationspartner, wie den Menschen oder die Maschine, angebotene Schnittstellen, als auch das Kommunikationsmedium sowie das eingesetzte Protokoll.

In Abläufen können zeitliche Aspekte eine wichtige Rolle spielen, so z. B. bei der Bearbeitung von Aufgaben oder dem Treffen einer Entscheidung. Zeitbehaftete Ablaufbeschreibungen in Prozessmodellen sind von daher auch ein wichtiges Element bei der Behandlung von Gerechtigkeitsaspekten.

Selbst-Adaptivität beschreibt die Fähigkeit eines Systems, sich aufgrund geänderter Umstände selbst anzupassen (Luckey und Engels 2013). Eine Anpassung eines Ablaufs kann dabei u. a. die Ablaufstruktur, verwendete Ressourcen oder zeitliche Vorgaben betreffen. Ferner können komplexe Aufgaben von kontextspezifischen Parametern abhängig sein, die z. B. in Anlehnung an die Erfahrung von Beschäftigten unterschiedlich interpretiert werden und zu alternativen Ausführungspfaden führen können. Das Modell einer Ablaufstruktur kann darüber hinaus nicht nur vordefinierte, statische Abläufe, sondern auch sogenannte ad-hoc-Abläufe enthalten, die sich beim Enactment den realen Abläufen der Beschäftigten anpassen können und so einen weiteren Grundbaustein für eine Realisierung eines Gerechtigkeitsempfindens bilden.

Die genannten Elemente einer Prozessmodellierungssprache können in der Phase (1) Entwurf \& Analyse verwendet werden, um neben der eigentlichen Prozessstruktur insbesondere auch Gerechtigkeitsaspekte zu modellieren. In den darauffolgenden Phasen (2) Konfiguration und (3) Enactment muss dann die verwendete Prozessausführungsumgebung mächtig genug sein - auch im Zusammenspiel mit an Cyber-Physical Devices beobachteten und gemessenen Werten - um z. B. kontext- und situationsspezifische Parameter im laufenden Betrieb zu berücksichtigen.

Durch eine derartige phasen-übergreifende und durchgängige Berücksichtigung von Gerechtigkeitsaspekten kann auf die Bedürfnisse von Beschäftigten geachtet werden und bei diesen eine hohe Akzeptanz erreicht werden.

\subsection{Szenario: Mensch-Roboter-Team}

Die in den beiden vorherigen Abschnitten beschriebenen Gerechtigkeits- und Prozessaspekte werden in diesem Abschnitt in Form eines Shared-Workspace-Szenarios in Anlehnung an (Gombolay et al. 2014) praktisch erläutert.

In einem mittelständischen Unternehmen gibt es trotz zunehmender Automatisierung einige Produkte, deren Endmontage vornehmlich manuell in Rahmen von 
Shared-Workspaces durchgeführt wird. Ein Shared-Workspace bezeichnet eine Arbeitsplatzumgebung, in denen menschliche Akteure und Maschinen interagieren. Dabei können verschiedene Funktionen wie z. B. die Assistenz in Abläufen, Aufgabenplanung oder Entscheidungsfindung durch beide Gruppen exklusiv oder kooperativ übernommen werden. An den einzelnen Arbeitsplatzumgebungen für die Endmontage wurde sich ferner für ein Mensch-Maschine-Team entschieden, das aus zwei menschlichen Akteuren (A1, A2) und einem Robotersystem (RS) besteht.

Die Teammitglieder haben die folgenden Fähigkeiten:

- A1, A2: Montage von Komponenten

- RS: Transport einzelner Komponenten

Ferner übernimmt das Robotersystem zusätzlich Planungsfunktionen, die sowohl die Reihenfolge einzelner Aufgaben optimal berechnet als auch die anschließende Zuweisung an die menschlichen Akteure vornimmt. Folglich tritt das Robotersystem als entscheidende und Arbeit zuweisende Instanz auf.

Im Rahmen der Wahrnehmung von menschlichen Akteuren ergeben sich hinsichtlich der Gerechtigkeitsaspekte in diesem Szenario verschiedene Gestaltungsmöglichkeiten in Bezug auf die Aktivitäten des Mensch-Roboter-Teams (siehe Tab. 16.1).

So kann die Durchführung der Arbeitsplanung durch ein Prozessmodell beschrieben werden, dessen Ablauf sowohl Präferenzen als auch Fähigkeiten der menschlichen Akteure berücksichtigt. Hierzu sind jedoch zunächst Profile notwendig, in denen wesentliche Eigenschaften der Teammitglieder hinsichtlich der Montageaufgaben enthalten sind. Diese Profile können die erforderlichen Kompetenzen und Erfahrungen für einzelne Arbeitsschritte, aber auch physische Eigenschaften enthalten. Eine für die Montage

Tab. 16.1 Gestaltungsmöglichkeit hinsichtlich verschiedener Gerechtigkeitsaspekte in Bezug zu Aktivitäten des Mensch-Roboter-Teams.

\begin{tabular}{l|l|l|l|l|l}
\hline & & $\begin{array}{l}\text { Distributive } \\
\text { Gerechtigkeit }\end{array}$ & $\begin{array}{l}\text { Prozedurale } \\
\text { Gerechtigkeit }\end{array}$ & $\begin{array}{l}\text { Interpersonale } \\
\text { Gerechtigkeit }\end{array}$ & $\begin{array}{l}\text { Informationale } \\
\text { Gerechtigkeit }\end{array}$ \\
\hline Robotersystem & $\begin{array}{l}\text { Arbeitspla- } \\
\text { nung durch- } \\
\text { führen }\end{array}$ & $\mathrm{X}$ & & & \\
\hline & $\begin{array}{l}\text { Aufgaben zu- } \\
\text { weisen }\end{array}$ & $\mathrm{X}$ & $\mathrm{X}$ & $\mathrm{X}$ \\
\hline Mensch & $\begin{array}{l}\text { Aufgaben ab- } \\
\text { lehnen }\end{array}$ & & $\mathrm{X}$ & & $\mathrm{X}$ \\
\hline & $\begin{array}{l}\text { Weiteres Feed- } \\
\text { back angeben }\end{array}$ & & $\mathrm{X}$ & & $\mathrm{X}$ \\
\hline
\end{tabular}


relevante physische Eigenschaft könnte die Frage sein, ob es sich bei dem menschlichen Akteur um einen Links- oder Rechtshänder handelt. Die Zuteilung von Aufgaben mit dem Erfordernis der Verwendung hoch spezialisierter Werkzeuge kann hiervon abhängig sein, wenn diese exklusiv nur für Links- oder Rechtshänder geeignet sind.

Wurde die Zuteilung von Aufgaben geplant, muss der Roboter dem menschlichen Akteur die Zuweisung mitteilen. Eine derartige Zuweisung sollte unter Berücksichtigung informationaler, distributiver und interpersonaler Gerechtigkeitsaspekte realisiert werden. So sollten in Bezug auf die informationale Gerechtigkeit relevante Informationen (zumindest auf Nachfrage) dargestellt werden, die zu der Zuweisung geführt haben. Die Begründungen können sich bspw. auf die aktuell gespeicherten Präferenzen des menschlichen Akteurs oder auch auf Indikatoren seiner Arbeitsgeschwindigkeit oder -qualität beziehen. Ferner ist zu erwarten, dass der Aspekt der distributiven Gerechtigkeit auf Basis eines in der Entwurfsphase ausgewählten Verteilungsprinzips realisiert wird. Derartige Verteilungsprinzipien sind etwa das Beitragsprinzip (je höher das Gehalt, desto mehr/schwierigere Arbeit muss zugewiesen werden), das Bedürfnisprinzip (jeder soll die Aufgaben erhalten, die er/sie haben möchte) oder das Gleichheitsprinzip (jeder soll exakt die gleichen Aufgaben in einem bestimmten Zeitraum bearbeiten).

Eine Zuweisung sollte, der prozeduralen Gerechtigkeit folgend, bei geeigneter Informationslage nachvollziehbar bzw. reproduzierbar sein. Darüber hinaus ist der Aspekt der interpersonalen Gerechtigkeit durch adäquate Gestaltung einer Benutzerschnittstelle mit respektvollen und höflichen Anweisungen zu tätigen.

Die Prozessunterstützung kann vorsehen, dass der menschliche Akteur eine ihm zugewiesene Aufgabe annimmt oder ablehnt. Unter Berücksichtigung einer bilateralen informationalen Gerechtigkeit gegenüber dem Robotersystem ist der menschliche Akteur auch in der Pflicht, bei einer abgelehnten Aufgabe den Grund der Ablehnung zu dokumentieren. Dies kann im Rahmen einer Anpassung des gespeicherten Profils des menschlichen Akteurs verwendet werden, damit bei zukünftigen Entscheidungen des Robotersystems geänderte Parameter bei der Entscheidung berücksichtigt werden können. Eine derartige Anpassbarkeit des Planungsprozesses stellt einen prozeduralen Gerechtigkeitsaspekt dar.

Neben geplanten Interaktionen in Form der Zuweisung einer Aufgabe kann es aber auch ungeplante Ereignisse im Team geben, die durch eine Mensch-Maschine-Interaktion berücksichtigt werden müssen. So sind vom Robotersystem nicht erfassbare realweltliche Umstände möglicherweise durch die komplexen Wahrnehmungsfähigkeiten eines der menschlichen Akteure erfassbar (Bächler et al., Kap. 3). Hierbei kann es sich z. B. um eine nicht vorhergesehene Störung handeln, die eine Unterbrechung des Montagebetriebs notwendig macht. Daher ist es hinsichtlich einer prozeduralen Gerechtigkeit in diesem Fall notwendig, dass dem Akteur die Möglichkeit zur Anpassung des Produktionsprozesses gegeben wird Dies betrifft auch Aspekte der informationalen Gerechtigkeit, da die Umstände für eine Anpassung dokumentiert werden sollten. 
Das zuvor geschilderte Szenario beschreibt die Möglichkeiten der Behandlung von Gerechtigkeitsaspekten in Entscheidungsprozessen. Einige Aspekte, wie die Darstellung aller für die Planungsentscheidung relevanten Daten (informational) sowie der Nachvollziehbarkeit des Entscheidungsprozesses (prozedural), werden ebenso durch Vermin et al. (Kap. 9) als eine der notwendigen Voraussetzungen für die Integration des Menschen in zukünftigen sozio-technischen Produktionssystemen genannt. Neben der in diesem Abschnitt beschriebenen Planungsfunktion eines Robotersystems lassen sich auch weitere Systeme identifizieren, für die die Wahrnehmung von Gerechtigkeitsaspekten bereits im Entwurf eine Rolle spielen kann. So zählen Bächler et al. (Kap. 3) pädagogische und ethische Aspekte von Assistenzsystemen auf, die ebenso wie die Berücksichtigung von Gerechtigkeitsaspekten eine grundlegende Rolle hinsichtlich der Akzeptanz und des Entwurfs von Assistenzsystemen spielen und für den Kontext der Digitalisierung von Produktions- und Dienstleistungsprozessen näher untersucht werden müssen.

\subsection{Zusammenfassung und Ausblick}

In diesem Kapitel wurde erläutert, dass die in Industrie 4.0-Anwendungen typischen Produktions- und Entscheidungsprozesse verschiedene Formen von Gerechtigkeit berücksichtigen müssen. Hierzu wurden Sprachelemente von Prozessmodellierungssprachen benannt, die für die Modellierung von Gerechtigkeitsaspekten relevant sind. An dem Beispiel eines halb-automatisierten Produktionssystems wurde aufgezeigt, welche Gerechtigkeitsaspekte an welcher Stelle relevant sind.

Diese integrierte, interdisziplinäre Betrachtung und Erforschung der Berücksichtigung von Gerechtigkeitsaspekten in der Modellierung und Realisierung von Industrie 4.0-Anwendungen ist eine der Fragestellungen, die im NRW Fortschrittskolleg „Gestaltung von flexiblen Arbeitswelten - Menschen-zentrierte Nutzung von Cyber-Physical Systems in Industrie 4.0“ behandelt werden.

Insgesamt werden in dem Fortschrittskolleg folgende Themenfelder bearbeitet:

1. Übergang zu menschen-zentrierten, flexiblen und adaptiven Arbeitsprozessen unter Berücksichtigung ihrer gesellschaftlichen Einbettung und organisationalen Gerechtigkeit.

2. Einsatz von lernenden, intelligenten Assistenzsystemen in Form von Cyber-Physical Devices.

3. Entwicklung eines anforderungsorientierten Systems-Engineering-Ansatzes als Grundlage für eine interdisziplinäre Zusammenarbeit.

4. Untersuchung der daraus resultierenden Änderungen in den beruflichen Anforderungen mit Konsequenzen für die Aus- und Weiterbildung und das Lernen am Arbeitsplatz. 
Das NRW Fortschrittskolleg wird von Wissenschaftlerinnen und Wissenschaftlern der Universitäten Paderborn und Bielefeld betrieben. Die beteiligten Fachrichtungen sind Psychologie, Soziologie, Pädagogik, Elektrotechnik, Maschinenbau, Wirtschaftswissenschaften und Informatik. Die im Rahmen von zehn Promotionsprojekten durchgeführten Forschungsarbeiten sind eingebettet in ein inter- und transdisziplinäres Umfeld. Die Forschungsprojekte werden von der Initiierung bis hin zur abschließenden Evaluation von den Partnern des transdisziplinären Umfelds begleitet; dies sind der Spitzencluster it's OWL, die Technologieberatungsstelle beim DGB NRW e. V., die IG Metall NRW und das Innovationsnetzwerk Energie Impuls OWL e. V., Näheres zum Fortschrittskolleg findet man unter http://pace.uni-paderborn.de/studienprogramme/ fsk-gfa.html.

\section{Literatur}

Bauer, W., Gerlach, S., Hämmerle, M., \& Strölin, T. (2014). Rule base for operative planning and control of flexible labour hours. World Congress, 19, 10760-10765.

Colquitt, J. A. (2001). On the dimensionality of organizational justice: A construct validation of a measure. Journal of Applied Psychology, 86(3), 386-400. https://doi. org/10.1037/0021-9010.86.3.386.

Colquitt, J. A., Conlon, D. E., Wesson, M. J., Porter, C. O. L. H., \& Yee Ng, K. (2001). Justice at the millenium: A meta-analytic review of 25 years of organizational justice research. Journal of Applied Psychology, 86(3), 425-445. https://doi.org/10.1037//0021-9010.86.3.425.

Colquitt, J. A., Scott, B. A., Rodell, J. B., Long, D. M., Zapata, C. P., Conlon, D. E., \& Wesson, M. J. (2013). Justice at the millennium, a decade later: A meta-analytic test of social exchange and affect-based perspectives. Journal of Applied Psychology, 98(2), 199-236. https://doi. org/10.1037/a0031757.

Colquitt, J. A., \& Zipay, K. P. (2015). Justice, fairness, and employee reactions. Annual Review of Organizational Psychology and Organizational Behavior, 2(1), 75-99. https://doi.org/10.1146/ annurev-orgpsych-032414-111457.

Gombolay, M. C., Gutierrez, R. A., Sturla, G. F., \& Shah, J. A. (2014). Decision-making authority, team efficiency and human worker satisfaction in mixed human-robot teams. In Dieter Fox, Lydia E. Kavraki \& Hanna Kurniawati (Hrsg.), Proceedings of the Robotics: Science and Systems X.

Großkopf, A., Decker, G., \& Weske, M. (2009). The process: Business process modeling using BPMN. Tampa, FL: Meghan-Kiffer Press.

Hacker, W. (1984). Psychologische Bewertung von Arbeitsgestaltungsmaßnahmen: Ziele und Bewertungsmaßstäbe. Berlin: Springer-Verlag.

Hacker, W. \& Richter, P. (1984). Psychische Fehlbeanspruchung: Psychische Ermüdung, Monotonie, Sättigung und Streß. Berlin: Springer-Verlag.

Kagermann, H., Lukas, W.-D., \& Wahlster, W. (2011). Industrie 4.0: Mit dem Internet der Dinge auf dem Weg zur 4. industriellen Revolution, VDI-Nachrichten.

Kleinjohann, B., Kleinjohann, L., \& Engels, G. (2013). Cyber-Physical Devices - Die Schnittstelle zwischen Cyberspace und realer Welt. IKT.NRW Schriftenreihe, Clustermanagement IKT.NRW, Bergische Universität Wuppertal. http://ikt.nrw.de/publikationen/. Zugegriffen: 15. Dez. 2015. 
Luckey, M., \& Engels, G. (2013). High-quality specification of self-adaptive software systems. In Proceedings of the 8th international symposium on software engineering for adaptive and selfmanaging systems. New York, NY, USA: ACM, SEAMS '13, S. 143-152.

Radhakrishnan, P., Subramanyan, S., \& Raju, V. (2008). CAD/CAM/CIM. New Age International.

Shank, D. B. (2012). Perceived justice and reactions to coercive computers. Sociological Forum, 27(2), 372-391. https://doi.org/10.1111/j.1573-7861.2012.01322.x.

Shank, D. B. (2014). Technology and emotions. In J. E. Stets \& J. H. Turner (Hrsg.), Handbook of the sociology of emotions: Volume II (S. 511-528). Dordrecht: Springer Netherlands (Handbooks of Sociology and Social Research).

Töniges, T., Ötting, S. K., Wrede, B., Maier, G. W., \& Sagerer, G. (2017). Human-state recognition for process adaptation in cyber-physical systems and perception of machine-fairness: A humancentered perspective. In H. Song, D. B. Rawat, S. Jeschke, \& C. Brecher (Hrsg.), Cyber-physical systems: Elsevier JAI (intelligent data-centric systems: Sensor collected intelligence).

van der Aalst, W. M. P., \& Weske, M. (2013). Reflections on a decade of interorganizational workflow research. In J. Bubenko, J. Krogstie, O. Pastor, B. Pernici, C. Rolland, \& A. Solvberg (Hrsg.), Seminal contributions to information systems engineering: 25 Years of CAiSE (S. 307313). Berlin: Springer-Verlag.

Weske, M. (2012). Business process management: Concepts, languages, architectures (2. Aufl.). Berlin Heidelberg: Springer-Verlag.

Open Access Dieses Kapitel wird unter der Creative Commons Namensnennung 4.0 International Lizenz (http://creativecommons.org/licenses/by/4.0/deed.de) veröffentlicht, welche die Nutzung, Vervielfältigung, Bearbeitung, Verbreitung und Wiedergabe in jeglichem Medium und Format erlaubt, sofern Sie den/die ursprünglichen Autor(en) und die Quelle ordnungsge-mäß nennen, einen Link zur Creative Commons Lizenz beifügen und angeben, ob Änderungen vorgenommen wurden.

Die in diesem Kapitel enthaltenen Bilder und sonstiges Drittmaterial unterliegen ebenfalls der genannten Creative Commons Lizenz, sofern sich aus der Abbildungslegende nichts anderes ergibt. Sofern das betreffende Material nicht unter der genannten Creative Commons Lizenz steht und die betreffende Handlung nicht nach gesetzlichen Vorschriften erlaubt ist, ist für die oben aufgeführten Weiterverwendungen des Materials die Einwilligung des jeweiligen Recht-einhabers einzuholen.

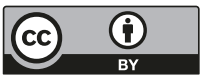

